\title{
Violencia cotidiana en la vida de las mujeres: Una aproximación al acoso sexual en espacios públicos costarricenses
}

\author{
Daily Violence in the Lives of Women: An Approach to Sexual Harassment \\ in Costa Rican Public Spaces
}

\section{Amanda Mesén Badilla}

\section{RESUMEN}

Este artículo reflexiona sobre los principales hallazgos en materia de acoso sexual en espacios públicos costarricenses, a la luz de las experiencias de mujeres que publicaron sus testimonios a través de la red social Facebook. Se realizó un estudio exploratorio basado en una metodología cualitativa. Se utilizó la técnica de análisis narrativo. Como principales resultados, se encontró que el acoso sexual en espacios públicos es una forma de violencia que ocasiona daños significativos en la subjetividad, organización de la vida y, cotidianidad de las mujeres. Existe riesgo de sufrir acoso sexual en espacios públicos solo por el hecho de acceder a ellos, sin embargo, algunas condiciones vulneran en mayor medida la seguridad de las víctimas. Además, el acoso sexual en espacios públicos es fundamentalmente una violación a los derechos humanos que impide el desarrollo pleno de la vida de las mujeres.

Palabras clave: Acoso sexual, espacios públicos, violencia contra las mujeres, feminismos, investigación, psicología.

\begin{abstract}
This article reflects on the main findings regarding sexual harassment in Costa Rican public spaces, based on the experiences of women who published their testimonies through the social network Facebook. An exploratory study based on a qualitative methodology was carried out. The narrative analysis technique was used. As the main results, it was found that sexual harassment in public spaces is a form of violence that causes significant damage to the subjectivity, organization of life and daily life of women. There is a risk of suffering sexual harassment in public spaces just for the fact of accessing them, however, some conditions further jeopardize the safety of the victims. Furthermore, sexual harassment in public spaces is fundamentally a violation of human rights that prevents the full development of women's lives.
\end{abstract}

Key Words: Sexual harassment, public spaces, violence against women, feminisms, research, psychology.

\footnotetext{
${ }^{1}$ Universidad de Costa Rica. San José, Costa Rica. Licenciada en psicología. Integrante del Proyecto Cero Tolerancia al Hostigamiento Sexual de la Universidad de Costa Rica. Correo electrónico: amanda.mesen.badilla@gmail.com

DOI: $10.15517 /$ WL.V1612.49219

Recepción: 20/02/2021

Aceptación: 2/11/2021
} 


\section{Introducción}

La lucha contra la violencia hacia las mujeres y sus múltiples manifestaciones, hoy, más de dos siglos después del inicio de los primeros movimientos feministas, está más vigente que nunca: El neoliberalismo se ha encargado de agudizar la feminización de la pobreza; un derecho tan básico como transitar en las vías públicas sigue siendo una actividad riesgosa en la vida de las mujeres; las denuncias por violencia doméstica siguen en aumento; y uno de los ejemplos más claros y desgarradores de los efectos de las estructuras de dominación y explotación, el femicidio, se ha vuelto un tema diario en los medios de comunicación.

En este sentido, según la Organización Mundial de la Salud (OMS) ${ }^{2}$ la violencia contra las mujeres es una violación de derechos humanos y un problema de salud pública que afecta a todos los niveles de la sociedad y causa consecuencias graves para la salud y el bienestar de quienes la sufren, información que revela la magnitud de esta problemática a nivel mundial.

En el caso del acoso sexual en espacios públicos, es importante considerar que es un tema de la vida diaria de las mujeres, y sobre esto conviene preguntarse, ¿cómo se configura la cotidianidad de las mujeres sabiendo que, en los espacios públicos, en cualquier momento y lugar pueden ser víctimas de violencia? ¿qué significa en sus vidas y cuerpos transitar en la calle con miedo? Para dar respuesta a lo anterior es necesario dar lugar a las experiencias de mujeres que han sido víctimas de acoso sexual en espacios públicos, de manera que se generen insumos para una mejor comprensión psicosocial de esta problemática que permitan además respaldar la exigencia de un compromiso desde el Estado y sus instituciones.

En los últimos años se ha ido despertando el interés en torno de esta problemática. Colectivas feministas han realizado grandes e importantes esfuerzos por visibilizarla e inclusive se ha logrado posicionar el tema en la agenda política del país. Sin embargo, se debe mencionar que, si bien a partir de estos avances significativos se empieza a reconocer

\footnotetext{
2 Organización Mundial de la Salud (OMS), “Informe año: 1999”, acceso: 14 de noviembre de 2020, www.opsecu.org
} 
el acoso sexual en espacios públicos como un tema a investigar, la información a nivel nacional es casi nula.

Este artículo se propone reflexionar sobre los principales hallazgos en materia de acoso sexual en espacios públicos costarricenses obtenidos en la elaboración de la tesis de licenciatura en Psicología de la Universidad de Costa Rica "Acoso sexual en espacios públicos. Análisis narrativo a partir de las experiencias vivenciadas por mujeres y publicadas en la red social Facebook"3. La investigación tuvo como objetivo principal analizar, desde las teorías feministas y desde el campo de la psicología política, las experiencias de mujeres que publicaron sus testimonios de acoso sexual en espacios públicos costarricenses a través de la red social Facebook.

\section{Metodología}

Este trabajo tiene un alcance exploratorio basado en una metodología cualitativa. Dicha elección está justificada siguiendo a Strauss y Corbin ${ }^{4}$ quienes proponen que la metodología cualitativa es una herramienta para obtener detalles complejos de algunos fenómenos como sentimientos, procesos de pensamiento y emociones. Esta afirmación resulta muy adecuada tomando en cuenta que en la pesquisa realizada se trabajó con las experiencias de las mujeres que han publicado sus testimonios de acoso sexual en espacios públicos.

Los criterios de inclusión utilizados para la selección de los relatos de las mujeres que escribieron sus testimonios de acoso sexual en espacios públicos fueron: ser mujer, haber vivido experiencias de acoso sexual en espacios públicos costarricenses, tener un relato de acoso sexual en espacios públicos en la página "Yo sufrí acoso callejero" de la red social Facebook y, ser mayor de 18 años.

\footnotetext{
3 Amanda Mesén, "Acoso sexual en espacios públicos. Análisis narrativo a partir de las experiencias vivenciadas por mujeres y publicadas en la red social Facebook" (Tesis de licenciatura, Universidad de Costa Rica, 2018).

${ }^{4}$ Anselm Strauss y Juliet Corbin. Bases de la investigación cualitativa. Técnicas y procedimientos para desarrollar la teoría fundamentada (Medellín: Editorial Universidad de Antioquia, 2002).
} 
Las etapas de recolección de información estuvieron divididas en tres momentos. El primer momento se realizó mediante la página de Facebook "Yo sufrí acoso callejero" de donde se obtuvo los testimonios de acoso sexual en espacios públicos costarricenses. El segundo momento consistió en hacer contacto con cuatro de las mujeres que publicaron su testimonio de acoso sexual en espacios públicos con el objetivo de hacer una entrevista para profundizar sobre el tema. El tercer momento se realizó una vez analizada la información de los relatos. Se contactó y propuso, a las cuatro mujeres que participaron en la entrevista a profundidad, la participación en un grupo de discusión para hablar sobre posibles propuestas para trabajar el tema del acoso sexual en espacios públicos a nivel de la respuesta del Estado.

Se utilizó la transcripción con el fin de llevar a cabo una sistematización detallada de toda la información. La técnica de análisis de los datos fue el análisis narrativo que, siguiendo a Riessman $^{5}$, resulta especialmente fructífero en el estudio de eventos vitales disruptivos, que pueden alterar la biografía, como podría representar una experiencia de violencia tal como lo es el acoso sexual en espacios públicos.

\section{Insumos teóricos para comprender el acoso sexual}

A pesar de que las conductas que constituyen el significado del acoso sexual han sido parte de la vida de las mujeres a lo largo de la historia, es hasta hace poco tiempo que es nombrado como tal y reconocido como una forma de violencia contra las mujeres. Según Sue Wise y Liz Stanley ${ }^{6}$ se debe tener presente que las definiciones son producto de complejos procesos sociales y su origen puede localizarse en las actividades de grupos e individuos concretos, que construyen con determinados propósitos.

En el caso del acoso sexual fueron las feministas estadounidenses quienes cerca del año 1974 se interesaron en analizar sus experiencias con hombres en el trabajo. Fue así como buscaron una expresión que nombrara las conductas masculinas que superficialmente aparentaban ser sexuales pero que de hecho constituían un ejercicio de poder. Para el año

\footnotetext{
${ }^{5}$ Catherine Riessman, C. Narrative analysis. (Newbury Park: Editorial Sage, 1993).

${ }^{6}$ Sue Wise y Liz Stanley, El acoso sexual en la vida cotidiana (Barcelona: Editorial Paidós, 1992), 45.
} 
1975 el término acoso sexual ya fue ampliamente utilizado como parte de la lucha contra la discriminación a las mujeres en el campo laboral ${ }^{7}$.

Un aporte de suma importancia al tema lo hace la investigadora Laura Guzmán quien distingue tres características principales que definen una conducta de acoso sexual ${ }^{8}$ :

1. Es una conducta sexual: siempre que se habla de acoso sexual, se refiere a una situación que tiene implícita o explícitamente un componente que apela a la sexualidad, a la intimidad, al cuerpo o al espacio privado de la persona afectada.

2. Es una conducta indeseada por quien la recibe: no toda conducta de naturaleza sexual puede tipificarse como acoso sexual, para que eso sea así se requiere de una condición: el rechazo de la conducta por parte de la persona que la recibe.

3. Provoca efectos perjudiciales en la persona que recibe la conducta: el acoso sexual al ser una conducta que no es deseada por quien la recibe, va a provocar efectos negativos en la víctima.

En el marco jurídico costarricense la ley 7476 contra el Hostigamiento Sexual en el Empleo y la Docencia ${ }^{9}$ fue aprobada el 14 de diciembre de 1994 y puesta en vigencia el 3 de marzo de 1995 en concordancia con las discusiones que se estaban dando en la agenda política a nivel mundial sobre el lugar de subordinación de las mujeres y las consecuencias que esto genera en sus vidas. El origen de esta ley se da a partir de la necesidad de proteger los principios constitucionales básicos del respeto por la libertad y la vida humana, el derecho al trabajo y el principio de igualdad ante la ley, obligando al Estado a condenar la discriminación por razón de sexo y a establecer políticas para eliminar la discriminación contra la mujer. Dentro de esta se define el hostigamiento sexual como “...toda conducta sexual indeseada por quien la recibe, reiterada y que provoque efectos perjudiciales en los siguientes casos:

a) Condiciones materiales de empleo o de docencia

b) Desempeño y cumplimiento laboral y educativo

\footnotetext{
${ }^{7}$ Wise y Stanley, El acoso sexual en la vida cotidiana, 72.

${ }^{8}$ Laura Guzmán, Informe Final: Proyecto de investigación-acción Prevención del Hostigamiento Sexual en la Universidad de Costa Rica (San José: Universidad de Costa Rica, 2005), 8.

${ }^{9}$ Ley contra el Hostigamiento Sexual en el Empleo y la Docencia n. ${ }^{\circ} 7476$.
} 
c) Estado general de bienestar personal

También se considera acoso sexual la conducta grave que, habiendo ocurrido una sola vez, perjudique a la víctima en cualquiera de los aspectos indicados"10. Asimismo, en marzo del año 2007 se presentó un Proyecto de Ley con el objetivo de solventar inconsistencias y algunas omisiones de la Ley 7476, publicado en la gaceta como la ley 8805 en el mes de junio del año $2010^{11}$.

\section{Acoso sexual en espacios públicos}

El acoso sexual en espacios públicos, como muchas otras formas de violencia contra las mujeres, es un problema invisible. Para Ana de Miguel comprender esta invisibilidad es comprender que para la mayoría se solapa con el orden normal y natural de las cosas ${ }^{12}$. Esto en parte porque hasta hace poco predominaba una explicación de corte biologista y psicologista que explicaba la violencia masculina como "algo natural" y en todos los casos extremos, como producto de patologías individuales ${ }^{13}$.

De esta forma, a pesar de que en la actualidad se ha dado un importante avance en cuanto al reconocimiento de esta problemática, todavía es necesario convencer a la mayoría de la población sobre su existencia. Debido a que nuestras prácticas cotidianas están tan permeadas de manifestaciones de violencia sexista existe una gran dificultad para identificarlas, aunque estén pasando frente a nuestros ojos o inclusive, cuando las víctimas somos nosotras mismas. Sobre esto Rita Segato ${ }^{14}$ aporta que a pesar de los cambios que se han dado con respecto al lugar de las mujeres en el mundo, se preservan las nomenclaturas y ocurre una falsa impresión de continuidad de la vieja ordenación, pero que es ahora regida por otra estructura. Ante la falta de claridad sobre los cambios ocurridos, las mujeres se someten sin saber cómo contestar a la frase "siempre fuimos así” y a su reivindicación de la manutención de una costumbre que suponen o afirman tradicional ${ }^{15}$. En el contexto

\footnotetext{
${ }^{10}$ Ley contra el Hostigamiento, artículo 3.

${ }^{11}$ Ley de modificación de la Ley contra el Hostigamiento Sexual en el Empleo y la docencia n. ${ }^{\circ} 8805$.

12 Ana de Miguel, Neoliberalismo Sexual (Madrid: Ediciones Cátedra, 2015), 68.

13 Ana de Miguel, Neoliberalismo Sexual, 70.

${ }^{14}$ Rita Segato, La guerra contra las mujeres (Madrid: Traficantes de sueños, 2016), 121.

${ }^{15}$ Rita Segato, La guerra contra las mujeres, 123.
} 
costarricense el acoso sexual en espacios públicos es difícilmente nombrado entre astutas tretas culturales que lo difuminan entre galantería e idiosincrasia, y acompañado de una escasa educación sexual sujeta al prejuicio para hablar abiertamente de la sexualidad, ante cualquier respuesta frente a esta forma de violencia las mujeres se topan con un recurso central en el sostenimiento de la invisibilidad: la falta de credibilidad ${ }^{16}$.

En suma, según Amanda Mesén ${ }^{17}$ el acoso sexual en espacios públicos es definido como una forma de violencia cotidiana contra las mujeres, llevada a cabo mayoritariamente por hombres desconocidos en espacios públicos, que genera consecuencias serias en los sentimientos, conductas, espacio, tiempo y, cuerpo de las víctimas. Asimismo, es una experiencia formativa constante que tiene un mensaje de poder y control claro: para las mujeres ingresar a los espacios públicos involucra correr el riesgo de ser víctimas de múltiples manifestaciones de violencia que en el caso de ser denunciadas las expondrá a ser víctimas de más violencia ${ }^{18}$.

\section{Manifestaciones del acoso sexual en espacios públicos:}

\section{dónde y cómo se lleva a cabo}

De acuerdo con lo encontrado en los relatos no existe un lugar específico dentro de los espacios públicos en el que las mujeres son víctimas de esta forma de violencia. Es decir que las mujeres están en riesgo de sufrir acoso sexual en espacios públicos solo por el hecho de acceder a ellos, independientemente del escenario específico al que se ingrese (calles, trasportes públicos, establecimientos públicos, espacios de recreo). Sin embargo, existen dos condiciones particulares que las mujeres asocian con un mayor riesgo de sufrir acoso sexual en espacios públicos: lugares solitarios y horarios nocturnos:

"Eh, yo soy súper tramada y pienso demasiado como salir a la calle sola en las noches principalmente, como que eso a mí me trama mucho, me da miedo caminar sola..."19

\footnotetext{
${ }^{16}$ Amanda Mesén, “Acoso sexual en espacios públicos...”, 91.

17 Amanda Mesén, "Acoso sexual en espacios públicos...”, 120.

${ }^{18}$ Amanda Mesén, “Acoso sexual en espacios públicos...”, 100.

${ }^{19}$ Comunicación personal, 4 de octubre del 2017.
} 
Para poder entender los resultados citados anteriormente es importante considerar que, dentro de la lógica del sistema de dominación masculina, lo público y lo privado ha estado establecido para hombres y mujeres respectivamente. Desde los juegos de niños y niñas donde los primeros tienen mayor acceso a ocupar el espacio público con actividades al aire libre, mientras las segundas deben permanecer en sus casas para protegerse de los peligros, hasta el mandato tan acatado de que las mujeres deben quedarse en la casa asumiendo el trabajo doméstico mientras los hombres trabajan fuera de ella, han consolidado y perpetuado la idea que asocia a los hombres con lo público y a las mujeres con lo privado.

Y es que si bien es cierto desde hace algunos años se ha dado un cambio en el acceso de las mujeres al espacio público, la violencia cotidiana a la que se ven expuestas al ingresar a ellos deja en evidencia que, en la práctica, estos espacios no les corresponden y con eso sus posibilidades de desarrollarse en lo público siguen siendo sumamente limitadas. Es decir, los espacios públicos no son iguales para los hombres y para las mujeres. De ahí la importancia de analizar no sólo dónde participan las mujeres, sino en qué condiciones lo hacen ${ }^{20}$.

Se podría decir entonces, que la entrada de las mujeres en lo público tiene la condición de que sus derechos y necesidades particulares se mantengan en lo privado. Esto a su vez desvincula las formas de violencia que sufren las mujeres en los espacios públicos de la escena de lo social, lo que da como resultado que no se hablan, y a pesar de que suceden en presencia de otras personas por lo general nadie se involucra. Lo anterior se refleja en el siguiente relato:

"Al acercarse, me agarró un seno con tanta violencia que todavía recuerdo el dolor. De la rabia empecé a gritar, junté la piedra más grande que pude del piso, pero el temblor de angustia de todo el cuerpo y mi brazo de 14 años no lograron alcanzarlo; apenas llegaron a que la piedra rebotara casi en sus pies. Lo seguí cuando intenté que la piedra lo alcanzara, gritando tanto y tantas cosas y con tanta angustia que varias personas de locales comerciales

\footnotetext{
${ }^{20}$ Virginia Vargas, Programa Regional: Ciudades sin Violencia hacia las Mujeres Ciudades Seguras para Todas y Todos (Buenos Aires: Fondo de Desarrollo de las Naciones Unidas para la Mujer, 2007), 36.
} 
sobre la acera de enfrente salieron a ver. Me vieron temblando, enojadísima, llorando... Nadie se metió". 21

La enorme carga de violencia ejercida sobre las mujeres no es accidental. Tal como sucede en los espacios privados, en los espacios públicos existe una articulación entre lo físico y las relaciones sociales, en las que lo político e ideológico tienen un papel determinante ${ }^{22}$.

Tomando en cuenta que la articulación de la historia, la ideología y las prácticas generadas en cada sociedad construyen el espacio, se puede decir que los espacios públicos no sólo contienen y expresan relaciones de dominación de hombres sobre mujeres, sino que son hegemónicamente masculinos ${ }^{23}$. El resultado de esto es que las prácticas que en ellos se generan están dentro de la lógica masculina del espacio, por lo que la violencia a la que están expuestas cotidianamente las mujeres es naturalizada, justificada e invisibilizada. De esta forma se construye una de las particularidades del acoso sexual en espacios públicos: es considerado inevitable, tal como se muestra en el siguiente relato:

"En el primer año de la U después de clases, iba caminando por Heredia a tomar el bus junto a mi amiga. Mientras cruzábamos la calle, apareció un tipo en bicicleta que tocó mis senos de forma brusca y se fue riéndose fuertemente... Después de esto, me acostumbré a andar en la calle ocultando mi pecho con el bolso, los cuadernos, el abrigo o lo que tuviese a mano para protegerme". ${ }^{24}$

En cuanto al cómo se dan los acontecimientos de acoso sexual en espacios públicos, las manifestaciones que se categorizan en los resultados de la investigación son: tocamientos; miradas y gestos; palabras, gritos, y sonidos; exhibicionismo; desde vehículos; amedrentamiento. Todas las anteriores tienen como elemento común que van dirigidas hacia el cuerpo de las mujeres.

\footnotetext{
${ }^{21}$ Comunicación personal, 10 de octubre del 2015.

${ }^{22}$ Henry Lefebvre, La producción del espacio (Madrid: Editorial Capitán Swing, 2013), 141.

${ }^{23}$ Amanda Mesén, "Acoso sexual en espacios públicos...," 21.

${ }^{24}$ Comunicación personal, 10 de octubre del 2015.
} 
En relación con la violencia ejercida sobre el cuerpo de las mujeres Rita Segato $^{25}$ define la violación como el uso y abuso del cuerpo del otro, sin que este participe con intención o voluntad comparadas. Además, plantea que esta puede darse de diferentes formas -no todas igualmente observables- entre ellas la violación alegórica, en la cual no se produce un contacto que pueda calificarse de sexual, pero hay intención de abuso y manipulación indeseada del otro ${ }^{26}$. A partir de la información anterior se puede entender el acoso sexual en espacios públicos como una forma de violación alegórica:

"Pasó un tipo y me tocó, o sea, yo sentí que me tocó hasta el ano, ¿me entendés? Yo brinqué y me quedé así, y volví a ver a todo lado, ni siquiera supe quien fue, solo supe que pasó, y eso, yo sentí mucha culpa porque yo dije: me separé de mi papá, esto no tenía que haberme pasado, si me hubiera quedado con él esto no pasa" (Comunicación personal, 6 de octubre del 2017).

A la luz de los planteamientos de Rita Segato $^{27}$ sobre el paso abrupto de la sociedad premoderna a la sociedad moderna y el proceso de implantación poco reflexiva de la modernidad que a su vez tiene como consecuencia el resurgimiento del derecho natural de apropiación del cuerpo femenino cuando se le percibe en condiciones de desprotección, se puede decir que con la entrada de las mujeres a lo público se da el entrecruzamiento de dos situaciones. Por un lado, el ingreso de las mujeres a los espacios públicos considerado como una irrupción al orden natural de las cosas, por otro lado, la necesidad de los hombres de adquirir y restaurar el estatus de masculinidad. Ambas situaciones tienen como punto de encuentro los cuerpos de las mujeres sobre los que se ejerce la violencia como acto confirmatorio de esta masculinidad. De esta forma queda en evidencia que, aunque lo sexual es el instrumento para llevar a cabo las manifestaciones de violencia, el acoso sexual en espacios públicos es un acto de control y poder ejercido sobre los cuerpos de las mujeres con repercusiones directas en la cotidianidad de sus vidas.

\footnotetext{
${ }^{25}$ Rita Segato, Las estructuras elementales de la violencia (Buenos Aires: Prometeo Libros, 2010), 22.

${ }^{26}$ Rita Segato, Las estructuras elementales de la violencia, 44.

${ }^{27}$ Rita Segato, La guerra contra las mujeres, 105.
} 


\section{Comprensión del acoso sexual en espacios públicos costarricenses}

El análisis de los relatos nos lleva a pensar que las mujeres caracterizan el acoso sexual en espacios públicos como experiencias negativas recurrentes, relacionadas a la invasión de sus cuerpos. No obstante, existe una gran dificultad para nombrar estas experiencias como manifestaciones de violencia, al mismo tiempo que se tiende a minimizar las consecuencias que estas han generado en sus vidas. Una explicación posible para este fenómeno se puede encontrar en la naturalización de esta forma de violencia.

"Creo que en una situación de agresión, ya como en un tipo de abuso físico es más probable que alguien se meta pero cuando es así como acoso, no creo". ${ }^{28}$

Para ahondar en lo anterior se hará uso del concepto "banalidad del mal" ${ }^{29}$ que hace referencia a las acciones dañinas que no tienen un motivo maligno latente, y que según Hannah Arendt tiene que ver con la pura y simple irreflexión. De acuerdo con Amanda Mesén $^{30}$, en el caso de las mujeres, vivir bajo la consigna de complacer al otro desapropiándose de sus vidas, involucra vivencias de su sexualidad repletas de violencia, dando como resultado la irreflexión del acoso sexual como forma de violencia entendiéndolo como una vivencia más de la sexualidad asumida como "normal" dentro del sistema patriarcal. En el mismo sentido, Pierre Bourdieu plantea el concepto violencia simbólica que hace referencia a la sumisión ni siquiera percibida como tal, apoyándose en un sistema de creencias socialmente inculcadas $^{31}$.

Un ejemplo que ilustra lo expuesto anteriormente se puede encontrar en el hecho de que las mujeres participantes de la investigación relacionan acontecimientos de acoso sexual en espacios públicos con eventos de iniciación de su vida sexual ("mi primer contacto con un miembro masculino", "la primera vez que me tocaron", "a mí nadie me había tocado, estaba

\footnotetext{
${ }^{28}$ Comunicación personal, 10 de octubre del 2017.

${ }^{29}$ Hannah Arendt, Eichmann en Jerusalén. Un estudio sobre la banalidad del mal (Barcelona: Editorial Lumen, 1999), 63.

${ }^{30}$ Amanda Mesén, “Acoso sexual en espacios públicos...”, 148.

${ }^{31}$ Pierre Bourdieu, La dominación masculina (Barcelona: Editorial Anagrama, 2013), 49.
} 
súper chiquitilla"). De este modo, no hay una distinción clara entre manifestaciones de violencia y vivencias de la sexualidad:

"Yo tuve una adolescencia demasiado inocente, digamos, o sea, yo nunca vi pornografía, nunca nada, entonces, ese fue mi primer contacto con un miembro masculino". ${ }^{32}$

\section{Impacto del acoso sexual en espacios públicos costarricenses}

Para hablar del impacto que tiene el acoso sexual en espacio públicos en la vida de las mujeres se hará referencia a dos momentos particulares que las víctimas experimentan cuando sufren alguna manifestación de este tipo de violencia: reacción inmediata e impacto. En cuanto a la reacción inmediata se identificó que las mujeres experimentan enojo, miedo, parálisis, humillación, culpa, asco y, vulneración; sobre el impacto se identificó miedo, enojo $\mathrm{y}$, huella de la violencia, entendiendo esta última como todos los acontecimientos de la vida cotidiana de las mujeres que estén determinados por un rastro de la violencia vivida por el acoso sexual en espacios públicos.

En suma, el acoso sexual en espacios públicos produce consecuencias serias en la vida de las mujeres. Para Susana Velázquez ${ }^{33}$ si bien el acoso parece ser una manifestación de violencia sexual menos brutal que otras, los efectos son igualmente intensos por el control intimidatorio y coercitivo que ejercen los hombres sobre las mujeres. En este mismo sentido, Rita Segato haciendo referencia a la violación alegórica plantea que esta forma de manipulación forzada del cuerpo del otro desencadena un sentimiento de terror y humillación idéntico al causado por una violación cruenta ${ }^{34}$.

"El mae se quita el casco y me dice muchas cosas asquerosas, "mami chúpeme esta..." (señalando sus partes íntimas) ... Y unas frases más que no se me han borrado pero que ni siquiera quiero escribir porque son ASQUEROSAS... Y si es grotesco escribir/leerlo escucharlo es angustiante, da miedo! Sola, quedé asustada y no pude hacer nada!!! (de lo que me arrepiento muchísimo) él nada más se puso su casco de nuevo y se fue". ${ }^{35}$

\footnotetext{
${ }^{32}$ Comunicación personal, 8 de octubre del 2017.

${ }^{33}$ Susana Velázquez, Violencias cotidianas, violencias de género (Buenos Aires: Editorial Paidós, 2003 ), 27.

${ }^{34}$ Rita Segato, Estructuras elementales de la violencia, 40.

${ }^{35}$ Comunicación personal, 15 de octubre del 2015.
} 
En este punto es importante mencionar el lugar que tiene el miedo en relación con el impacto del acoso sexual en espacios públicos. De acuerdo con Virginia Vargas el cuerpo, particularmente el de las mujeres, aparece como depositario de las biografías, porque el cuerpo en sus tiempos, en sus espacios de violencia actuales e históricos, guarda memoria ${ }^{36}$. A la luz de los relatos se puede confirmar lo que plantea Susana Velázquez ${ }^{37}$, quien plantea que las mujeres desde niñas crecen sabiendo que hay algo de lo que deben protegerse, muchas veces a través de la advertencia de otras mujeres. Esto a su vez tiene un impacto directo en el proceso de socialización de las niñas que desde muy pequeñas cargan con el temor de "lo que podría pasar" o "lo que ha pasado a otras" que muy pronto se convierte en sus propias vivencias.

De esta forma, el miedo que experimentan las mujeres como consecuencia del acoso sexual en espacios públicos no solo aparece en el momento en el que se es víctima de algún acontecimiento de esta forma de violencia. El imaginario presente desde muy corta edad de "lo que podría pasar" genera un temor mucho menos evidente que a su vez origina consecuencias muy perjudiciales en el diario vivir de las mujeres, esto debido a que en apariencia no se muestra como una forma de control o prohibición explícita, sino que se presenta como una renuncia personal de las mujeres, provocando que se acepte lo cotidiano como lo que se acostumbra: cambiar de rutas, vestir de ciertas formas cuando se transita por las vías públicas, dejar de usar ciertos medios de transporte en horarios determinados, etc.

Por último, conviene destacar la articulación de uno de los mitos más difundidos en la cultura machista "que todo lo que les sucede a las mujeres es culpa de las mujeres", con los relatos de estas sobre la forma en la que el acoso sexual en espacios públicos les afecta. Para las mujeres ser víctimas de esta forma de violencia les genera una doble culpa: en un primer momento la culpa por el acoso recibido y, posteriormente la culpa por su accionar, independientemente de cuál haya sido su reacción, tal como se ejemplifica en el siguiente relato:

\footnotetext{
${ }^{36}$ Virginia Vargas, Programa Regional: Ciudades sin Violencia hacia las Mujeres Ciudades, 46.

${ }^{37}$ Susana Velázquez, Violencias cotidianas, violencias de género.
} 
"Recuerdo que me quedé paralizada y mi amiga me preguntó si estaba bien; le dije que "si" pero me fui a mi casa sintiéndome muy avergonzada, fea y tonta por no defenderme o gritar". 38

Todo lo anterior deja en evidencia el peso que tiene esta forma de violencia en el diario vivir de las mujeres. Desde el momento en el que planean las estrategias que utilizarán antes de salir de sus casas (vestuario, rutas, transporte, gestos, posibles respuestas). Sumado a esto, la humillación, angustia y miedo experimentado al enfrentar nuevamente y, a pesar de las estrategias previas, situaciones de acoso. El malestar de tener que lidiar con las responsabilidades y actividades del día en los distintos ámbitos de la vida (laborales, educativos, ocio, familiares, etc.) como si "nada hubiera pasado". Y un regreso rodeado de dolor e impotencia ante lo que se torna casi inevitable.

\section{Conclusiones}

A continuación, se presentan algunas de las principales conclusiones y desafíos por abordar a la luz de los hallazgos de la investigación.

El acoso sexual en espacios públicos es fundamentalmente una violación a la dignidad humana que impide el desarrollo pleno de la vida de las mujeres. Por sus características: manifiesta un mensaje claro sobre el lugar que le corresponde a las mujeres en la sociedad, no tiene un reconocimiento como problema social, es recurrente, se considera una práctica inevitable, atenta contra el bienestar de la vida de las mujeres; se puede decir que representa uno de los ejemplos más claros del espectro del sexismo en la sociedad costarricense.

Tomando en cuenta que esta forma de violencia tiene como trasfondo las relaciones desiguales de poder entre hombres y mujeres ejercidas por el sistema patriarcal, mientras no se redefina el lugar de las mujeres en los espacios públicos y privados, es decir, las imposiciones binarias de las identidades masculinas y femeninas, a pesar de que las mujeres amplíen su campo de acción, las dinámicas de violencia se seguirán reproduciendo en ellos. De acuerdo con lo anterior, es importante recalcar que crear una ruptura con el sistema de creencias que invisibiliza y justifica la violencia contra las mujeres involucra una reflexión

\footnotetext{
${ }^{38}$ Comunicación personal, 10 de octubre del 2015.
} 
sobre la condición de opresión en la que se vive y, el propio compromiso con el sistema que oprime.

De acuerdo con lo encontrado en los testimonios se identifican algunos desafíos por resolver en el camino por asegurar el derecho de las mujeres a ocupar la escena de lo público sin ser violentadas:

- La implementación de políticas integrales que garanticen la protección inmediata de las mujeres. Para esto es necesaria la articulación de distintos ámbitos de acción: educativo, medios de comunicación, arte y cultura, sensibilización de la institucionalidad democrática, etc.

- La obligatoriedad de los programas de educación sexual en los centros educativos desde el nivel de preescolar.

- Crear campañas masivas de información y prevención del tema dirigida a hombres y mujeres que apelen a la visibilización y sensibilización.

- Velar por el fortalecimiento de espacios seguros para que las mujeres puedan hablar del acoso sexual en espacios públicos y contar con los recursos para elaborar las experiencias vividas.

- Respaldar los importantes aportes que las mujeres, las colectivas, y demás agrupaciones como la colectiva Caminando, Peras del Olmo, y la asociación ciudadana ACCEDER han llevado a cabo en la visibilización del acoso como forma de violencia ${ }^{39}$.

- Con el objetivo de promover más trabajo conceptual, investigación y recopilación de datos, que permitan entender esta problemática, es necesario articular el trabajo de los activismos y academia de tal forma que se dé el reconocimiento del acoso sexual en espacios públicos como una forma de violencia contra las mujeres, otorgándole el lugar que le amerita dentro de los discursos legales y sociales.

\footnotetext{
${ }^{39}$ Las organizaciones mencionadas son colectivas feministas costarricenses que han tenido un papel central en el trabajo de visibilización de la problemática del acoso sexual en espacios públicos, entre otros temas de gran relevancia en la lucha por la defensa de los derechos de las mujeres en el país.
} 


\section{Referencias Bibliográficas}

Arendt, Hannah. 1999. Eichmann en Jerusalén. Un estudio sobre la banalidad del mal. Barcelona: Editorial Lumen.

Bourdieu, Pierre. 2013. La dominación masculina. Barcelona: Editorial Anagrama.

De Miguel, Ana. 2015. Neoliberalismo Sexual. Madrid: Ediciones Cátedra.

Guzmán, Laura. Informe Final: Proyecto de investigación-acción Prevención del Hostigamiento Sexual en la Universidad de Costa Rica. San José: Universidad de Costa Rica, 2005.

Lefebvre, Henry. 2013. La producción del espacio. Madrid: Editorial Capitán Swing.

Ley n. 7476 del 3 de marzo de 1995 contra el Hostigamiento Sexual en el Empleo y la Docencia.

Ley n. ${ }^{\circ} 8805$ de junio del 2010 de modificación de la Ley contra el Hostigamiento Sexual en el Empleo y la Docencia.

Mesén, Amanda. "Acoso sexual en espacios públicos. Análisis narrativo a partir de las experiencias vivenciadas por mujeres y publicadas en la red social Facebook". Tesis de licenciatura, Universidad de Costa Rica, San José, C.R. 2018.

Organización Mundial de la Salud. Informe año: 1999. Acceso: 14 de noviembre de 2020, www.opsecu.org

Riessman, C. (1993). Narrative analysis. Newbury Park: Editorial Sage.

Segato, Rita. 2016. La guerra contra las mujeres. Madrid: Traficantes de sueños.

Segato, Rita. 2010. Las estructuras elementales de la violencia. Buenos Aires: Prometeo Libros.

Strauss, A., Corbin, J. (2002). Bases de la investigación cualitativa. Técnicas y procedimientos para desarrollar la teoría fundamentada. Medellín: Editorial Universidad de Antioquia. 
(9) WIMB LU

Wimblu, Rev. Estud. de Psicología UCR, 16(2) 2021 (Julio-Diciembre): 161-177 /ISSN: 1659-2107

Vargas, Virginia. 2007. Programa Regional: Ciudades sin Violencia hacia las Mujeres Ciudades Seguras para Todas y Todos. Buenos Aires: Fondo de Desarrollo de las Naciones Unidas para la Mujer.

Velázquez, Susana. 2003. Violencias cotidianas, violencias de género. Buenos Aires: Editorial Paidós.

Wise, Sue y Stanley, Liz. 1992. El acoso sexual en la vida cotidiana. Barcelona: Editorial Paidós. 